\title{
Immediate Implant Placement in Extraction Socket with an Adapted Implant The New Astra Tech Implant EV in Routine Clinical Practice
}

\author{
Helmut G Steveling ${ }^{1 *}$, and José de San José González ${ }^{2}$ \\ ${ }^{1}$ Specialist in oral surgery, Main focus on implantology, Kelterbergstraße Gernsbach, Germany \\ ${ }^{2}$ General Dentistry, Germany
}

*Corresponding author: Helmut G Steveling, Specialist in oral surgery, Main focus on implantology, Kelterbergstraße Gernsbach, Germany, E-mail: helmut.steveling@t-online.de

Received: 27 Mar, 2020 | Accepted: 05 May, 2020 | Published: 11 May, 2020

Citation: Steveling HG, de San José González J (2020) Immediate Implant Placement in Extraction Socket with an Adapted Implant. Int J Dent Oral Health 6(4): dx.doi.org/10.16966/2378-7090.324

Copyright: (c) 2020 Steveling HG, et al. This is an open-access article distributed under the terms of the Creative Commons Attribution License, which permits unrestricted use, distribution, and reproduction in any medium, provided the original author and source are credited.

\section{Introduction}

Immediate implant placement in a fresh extraction socket is a proven and scientifically well documented treatment option. The implant system is a key to success, together with clinical and anatomical requirements and the experience of the surgeon. The new Astra Tech Implant EV has been optimized and adapted to meet the requirements of immediate implant placement. Using a patient case, the authors present specifications and special features of the procedure, as well as its execution in practice.

Routine clinical practice in implant dentistry requires procedures that are safe while also as efficient as possible. Today, this balancing act can be achieved using the immediate implant placement protocol. Installing an implant in a fresh extraction socket is a clinically proven and scientifically documented procedure with which good esthetic results and predictable long-term success can be achieved [1-4]. The procedure offers numerous advantages, such as minimally invasive access to the alveolus without flap debridement (blood supply is retained, no scarring, etc.), optimal support of the surrounding tissue (preservation of alveolar bone height and width), and a reduced number of treatment steps (high patient comfort).

However, especially in the sensitive anterior tooth area, the decision to perform a technically demanding immediate implant placement requires precise diagnosis and a rigorous assessment of all risks. The prerequisites are an inflammation-free alveolar cavity, an intact facial bone lamella, a stable soft tissue situation, a suitably experienced surgeon, and an appropriate implant system. One such system is the Astra Tech Implant System EV with its new Astra Tech Implant EV (Dentsply Sirona), which has been specifically adapted for immediate implant placement.

\section{Optimized Thread Geometry for Better Primary Stability}

Implant systems are continually being revised to ensure long-term success without complications. The Astra Tech Implant EV has been optimized to meet the specific requirement of ensuring primary stability in fresh extraction sockets and underprepared sites [5]. This was achieved by deepening the threads on the apical portion of the implant body (Figure 1). As the rest of the implant is unaffected by this adaptation, the drilling protocol remains the same. In the clinic and in the laboratory, a proven approach is followed using a known protocol.

For clinicians, the main advantage of the adapted implant is in its handling during the insertion. The tapering of the apical portion and the deeper thread profile make it easier to install the implant in extraction sockets and underprepared sites. In the residual basal bone, improved primary stability is attained that would otherwise be difficult to achieve given the lack of contact with the extraction socket walls.

\section{BioManagement Complex}

All known attributes of Astra Tech EV remain Implant System unchanged. The Astra Tech Implant EV continues to utilize:

- the well-documented OsseoSpeed surface [6-8] (chemically modified titanium surface for improved osseointegration);

- the reliable conical seal design $[9,10]$ (conical, self-centering implant abutment connection for a precise fit);

- the tried-and-true Microthread [11,12] (microthread on the implant collar for biomechanical bone stimulation); and

- the sophisticated connective contour $[13,14]$ (constriction of the abutment in the area of the implant collar to protect the marginal bone).

The outer dimensions of the entire implant-apart from the apical portion-are still the same. 


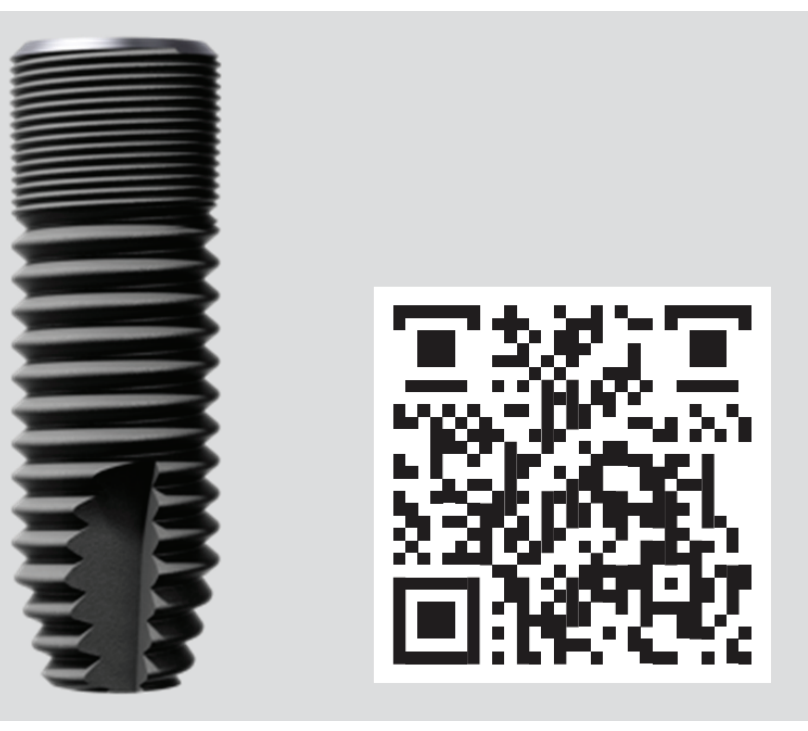

Figure 1: The new Astra Tech Implant EV with a deeper thread design in the apical area of the implant.

\section{Patient Case: In Practice}

\section{Initial situation}

A patient attended the clinic with a fractured tooth 12 . He reported that he had fractured his tooth in an accident. An X-ray diagnosis confirmed the initial suspicion of a fracture (Figure 2). A transverse fracture of the root of tooth 12 was diagnosed. In such cases, the main options are attempting to preserve the tooth or extracting it; the mere diagnosis of fracture does not preclude tooth preservation.

A crucial factor in deciding on a treatment is the path of the fracture line. In the case of a longitudinal fracture of the root, tooth preservation appears impossible. Similarly, in a transverse fracture, it is extremely difficult to preserve the tooth permanently using restorative endodontic measure

\section{Therapeutic decision}

Given the path of the fracture line on tooth 12 (deep transverse fracture), the predictability of tooth preservation was deemed low in this case, so extraction with immediate implant placement was considered. There are a variety of arguments in support of immediate implant placement. For example, extensive bone resorption and substantial receding of soft tissue in the area of the extraction socket can be prevented effectively. The flap-free procedure means that surgical trauma is low, the advantages of which include an enhanced esthetic result (no scarring). Rapid prosthetic rehabilitation and the reduced number of treatment sessions also make this treatment protocol an attractive option.

In this instance, all preconditions for immediate implant placement were met:

- Inflammation-free extraction socket

- Intact facial bone lamella and intact bony socket

- $\quad$ Stable soft-tissue situation

Attempted tooth preservation $v$ s. immediate implant placement: to obtain the best possible result, it was decided to extract the tooth and

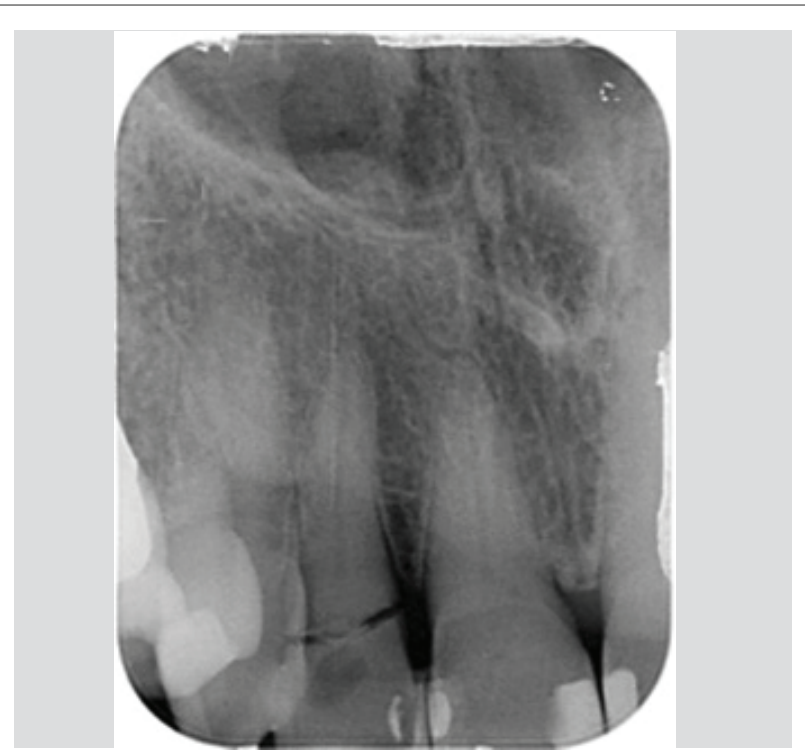

Figure 2: Transverse root fracture of tooth 12.

perform an immediate implant placement. Achieving the necessary primary stability is an important prerequisite for the success of immediate implant placement. For fresh extraction sockets, torques of more than $30 \mathrm{Ncm}$ is described in the literature [15]. The Astra Tech Implant EV, with its deeper thread design in the apical portion, was adapted to meet this requirement and was therefore the preferred choice in this case.

\section{Surgical procedure}

The fractured tooth 12 was carefully extracted while conserving as much of the alveolar walls as possible (Figure 3), and the tooth crown was separated from the root and retained for the temporary restoration. The implant was positioned palatally during insertion. A distance of around $1.5 \mathrm{~mm}$ from the adjacent teeth and around 2.5 $\mathrm{mm}$ from the buccal lamellae was maintained. The Astra Tech Implant EV (diameter $3.6 \mathrm{~mm}$, length $11 \mathrm{~mm}$ ) was inserted using the standard drilling protocol (Figure 4). The "self-cutting function" of the implant is strengthened by the deeper thread profile in the apical area, enabling its insertion into the extraction socket with comparative ease and little pressure (Figure 5). The gap to the facial bone lamella (jumping gap) resulting from the palatal orientation was not filled with augmentative materials. Experience has shown that bleeding into the gap appears to be a good approach for an immediate implant placement. The implant was temporarily sealed with a healing abutment (diameter $5.0 \mathrm{~mm}$, height $3.5 \mathrm{~mm}$ ) (Figure 6).

\section{Temporary restoration}

One day after the surgery, the wound was inspected and the first provisional treatment was performed by preparing the tooth 12 crown accordingly and splinting it to adjacent teeth 11 and 13 with composite (Figure 7). On inspection after four weeks of healing, the tissue was free of irritation and the gingiva was stable. A further four weeks later, the temporarily attached tooth fragment was removed and the scan post was placed on the implant in region 12 for digital intraoral scanning (Figures 8 and 9). There was a well-formed emergence profile at this time. After installation of the scan post, removal of saliva from the surrounding dentition and application of scan powder, the situation 


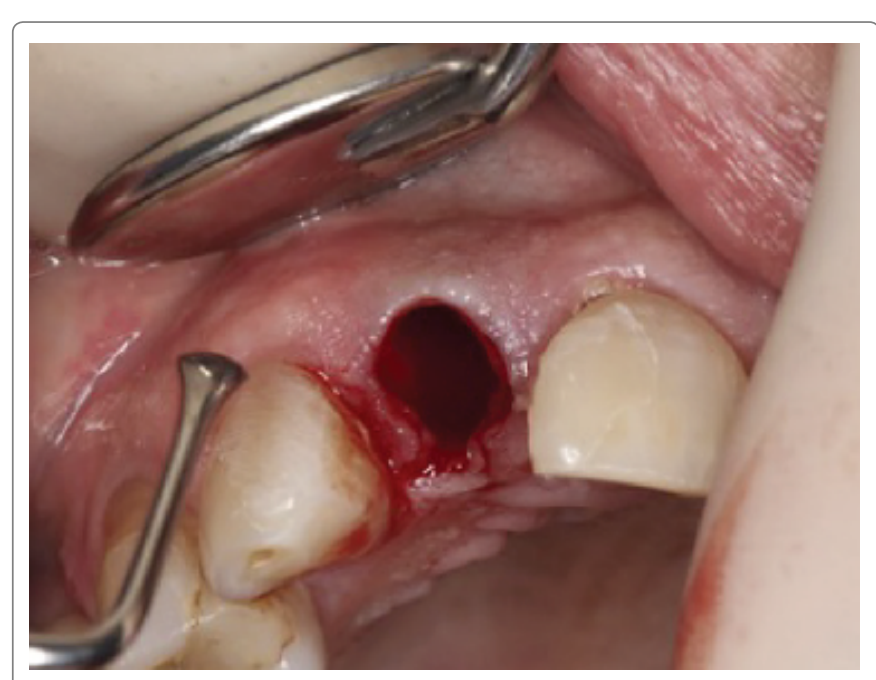

Figure 3: Intact socket after tooth 12 was carefully extracted.

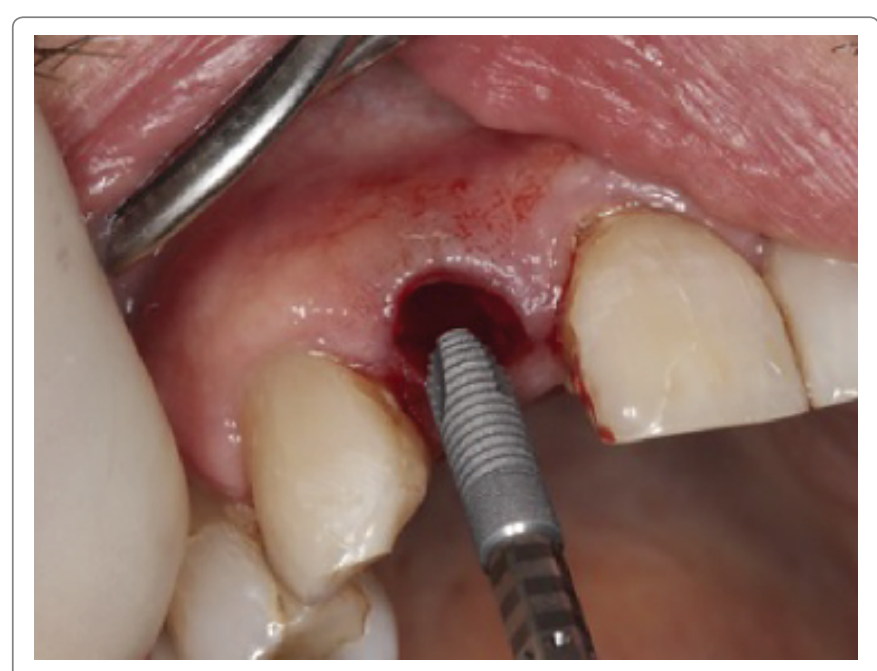

Figure 4: Insertion of the Astra Tech Implant EV into the fresh extraction socket.

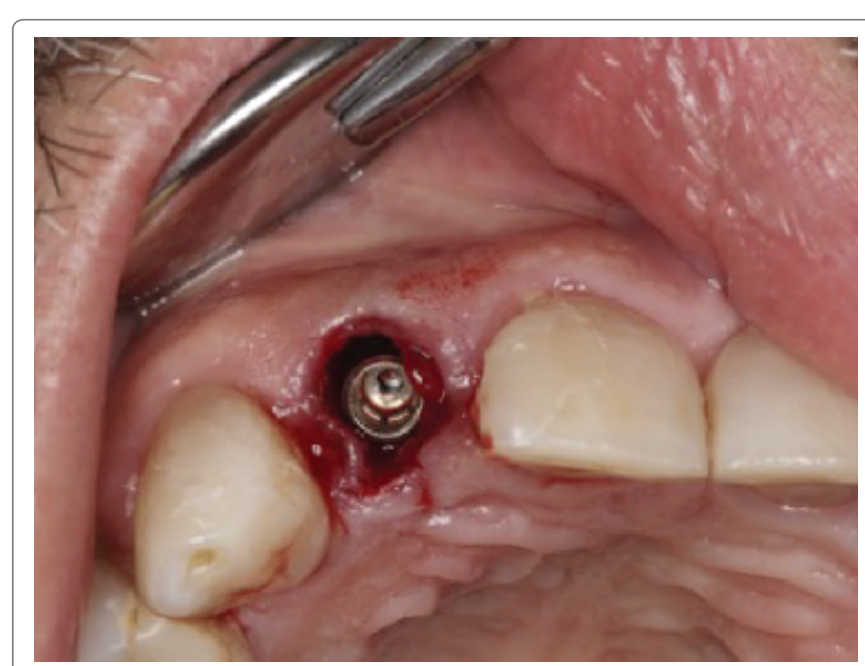

Figure 5: Primary stability achieved. The implant in its final position (three-dimensional orientation).

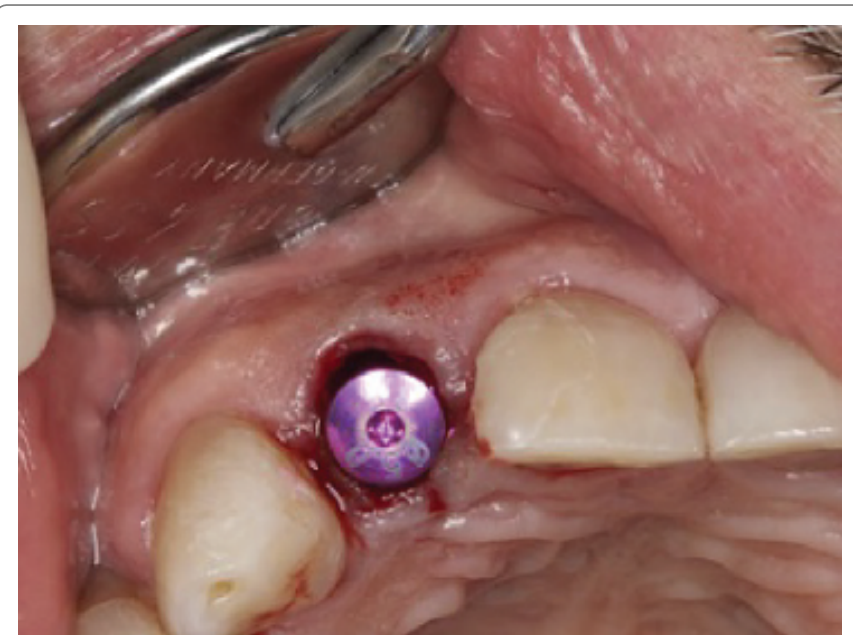

Figure 6: Coverage of the implant with a healing abutment.
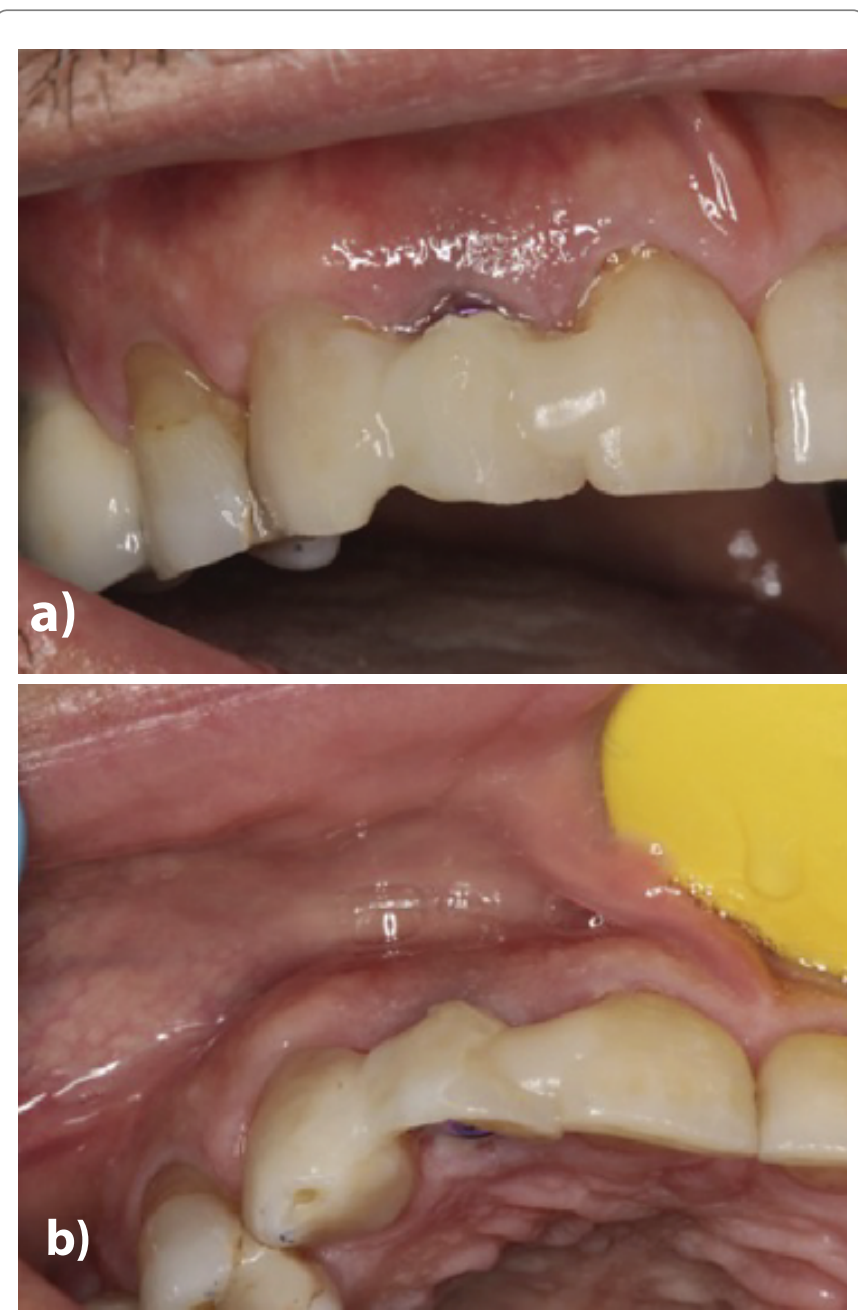

Figure 7: a) Coverage of the implant with a healing abutment.

b) Tooth fragment 12 (crown) was splinted to the adjacent teeth using composite. 

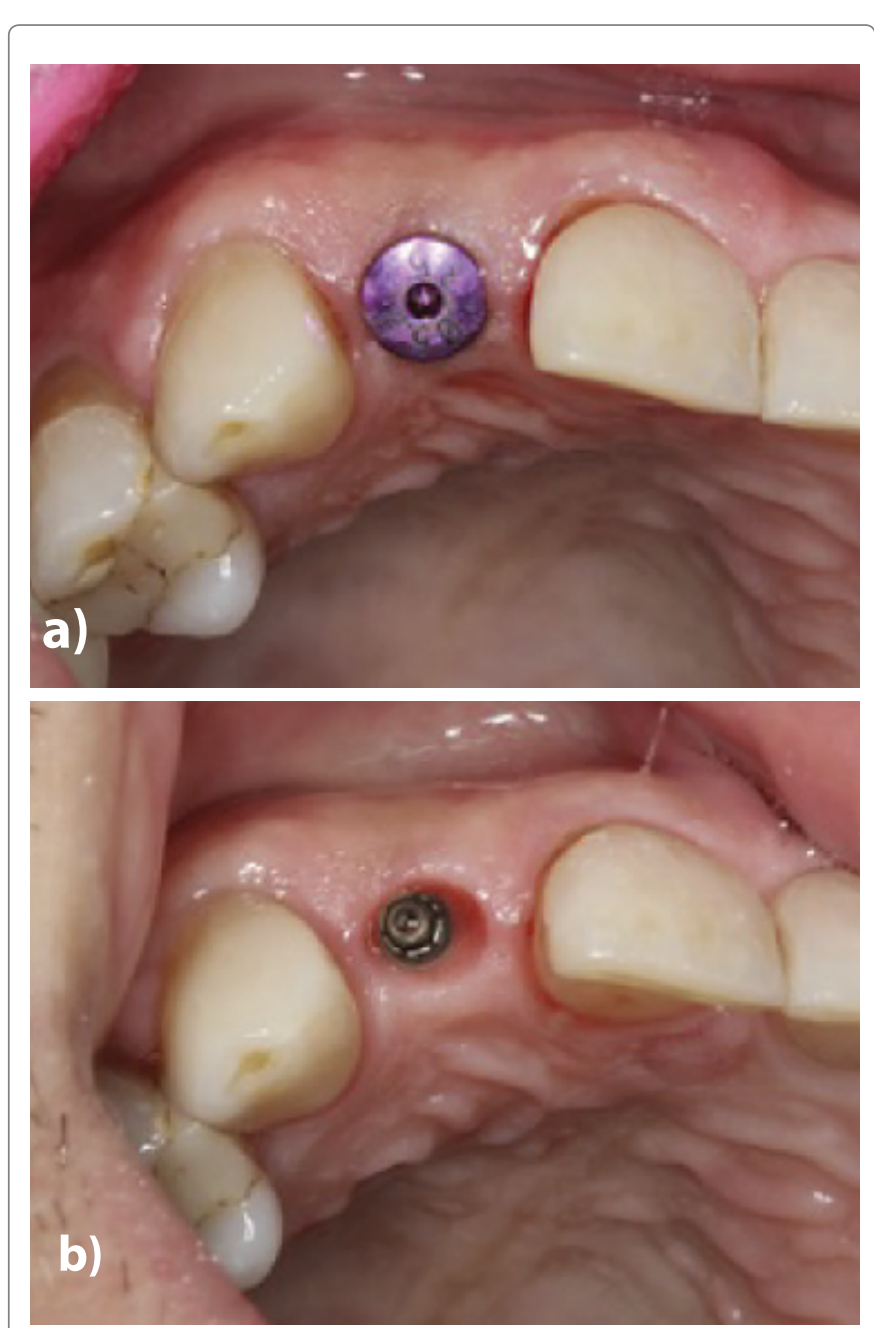

Figure 8: a) Eight weeks after insertion.

b) Removal of the temporary restoration.

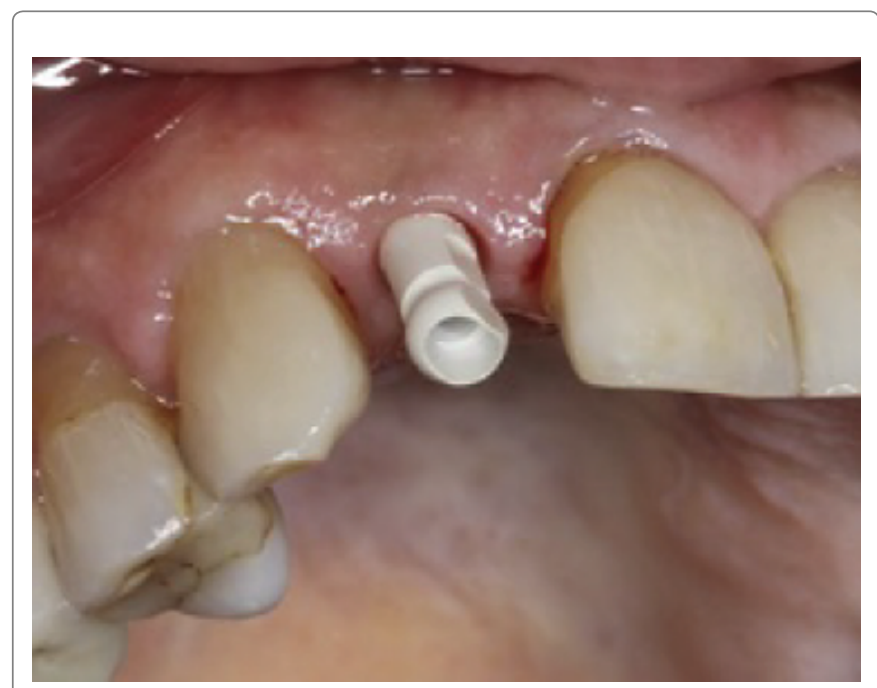

Figure 9: Placement of the scan post for intraoral digital scanning. was scanned with the intraoral scanner (True Definition, 3M) and the data sent to the laboratory. For the simplest possible temporary restoration, a one-piece abutment (Direct Abutment EV) was screwed onto the implant (Figure 10) and the corresponding healing cap was glued to a prefabricated tooth (Figure 11). The healing cap with ready-made tooth could then be affixed to the abutment with precise positioning by clicking it into place.

In contrast to a planned immediate implant placement with (temporary) immediate restoration, it was not possible to make full use of the digital workflow here following the tooth fracture because urgent action was required. If an implant placement can be planned, an individual Atlantis abutment can be ordered for exposure or immediate loading as part of the digital workflow. The Direct Abutment is available in different heights and diameters, and its shape is similar to a prepared tooth. The prefabricated abutment is suitable for the temporary restoration of non-angled implants. The corresponding healing cap, which snaps onto the abutment, can initially be used directly as a gingival former.

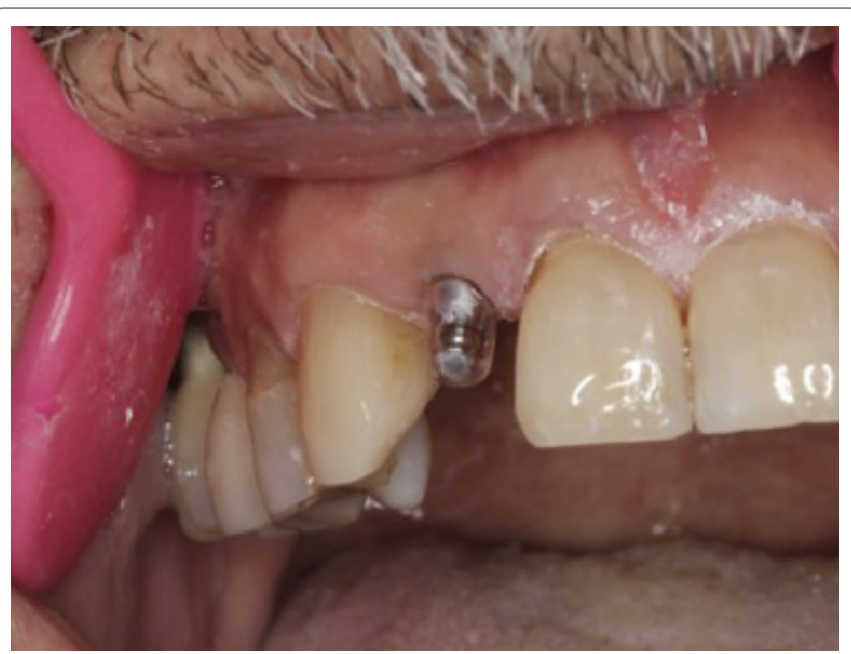

Figure 10: One-piece abutment (Direct Abutment EV) as the foundation for temporary restoration.

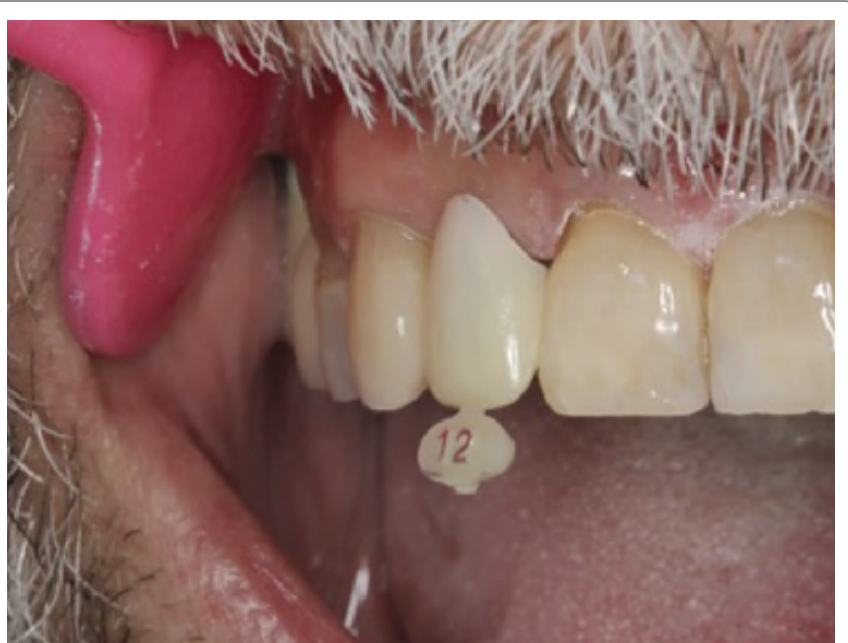

Figure 11: Prefabricated tooth to be glued (extra orally) to a healing cap. 


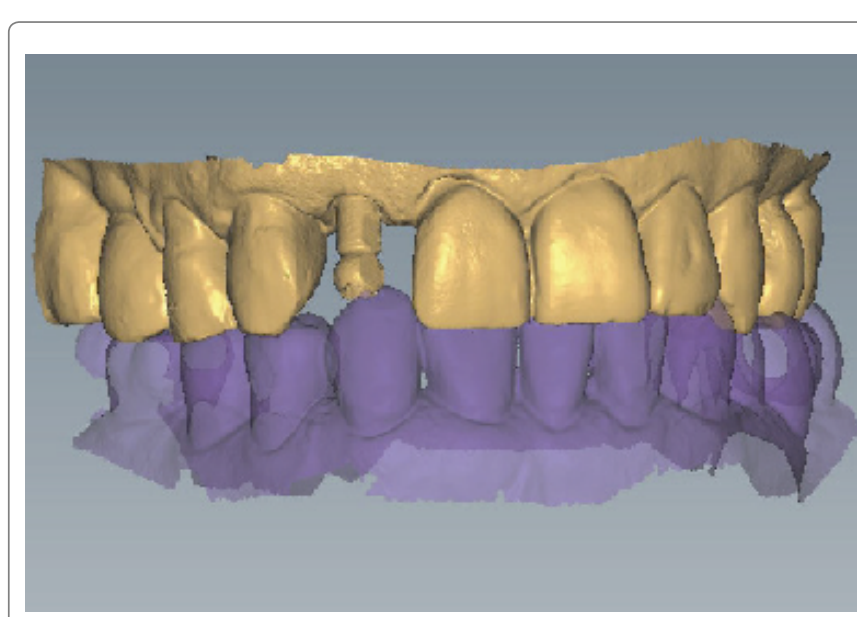

Figure 12: Representation of the digital impression in the Virtual Atlantis Design (VAD) software.

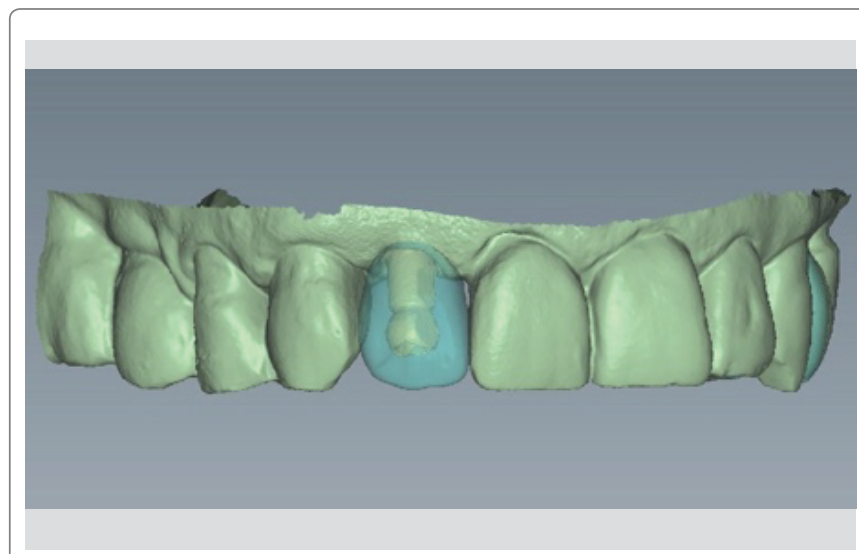

Figure 13: Preparation of the wax-up based on the digital data.

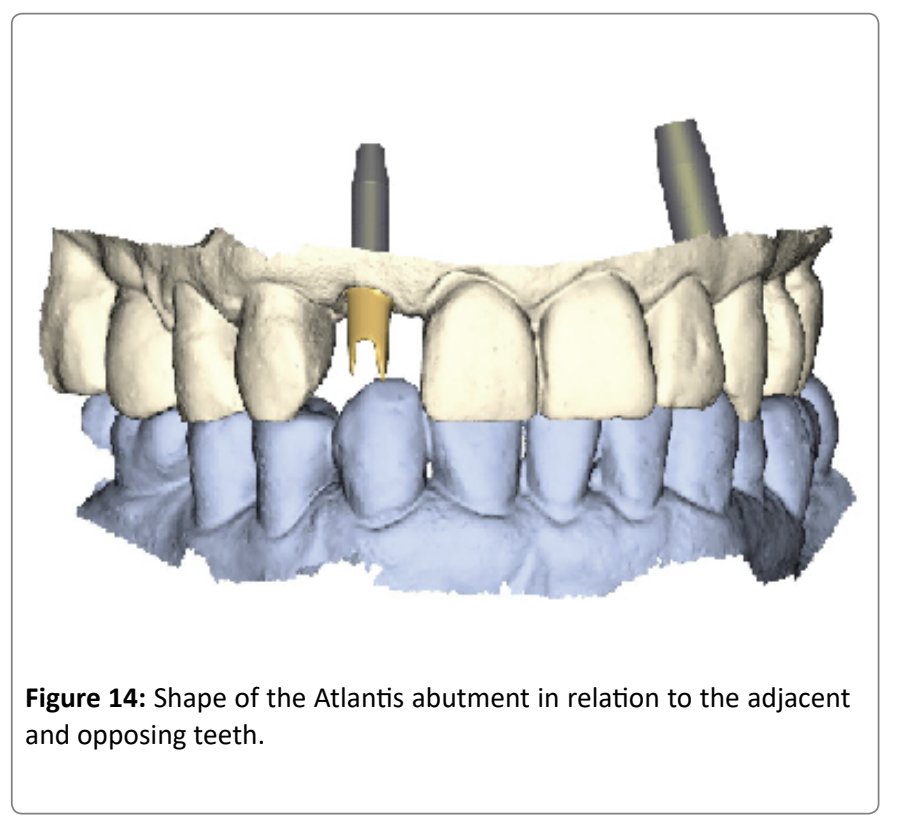

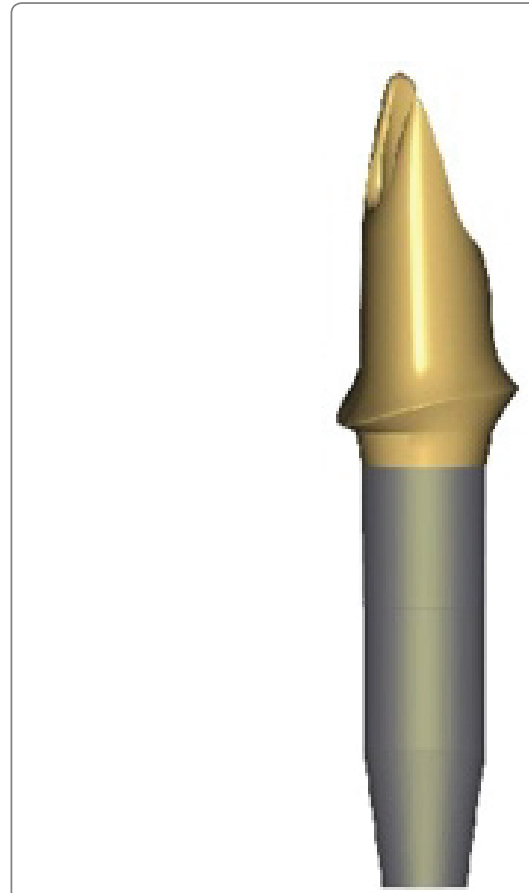

Figure 15: Gold-shaded Atlantis abutment.

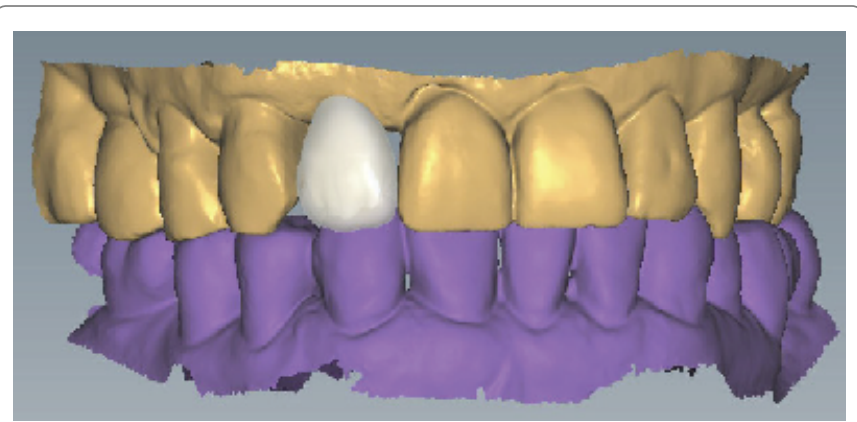

Figure 16: Design of the crown in the laboratory's proprietary CAD software.

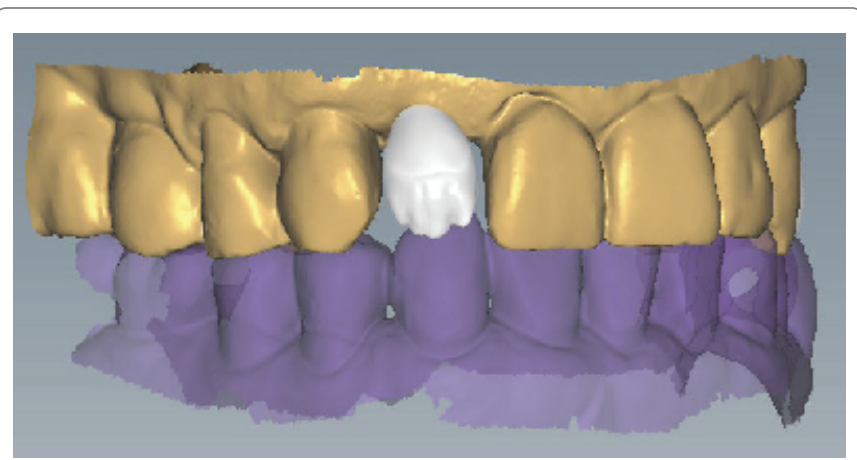

Figure 17: Design of the cut-back zirconium oxide crown. 


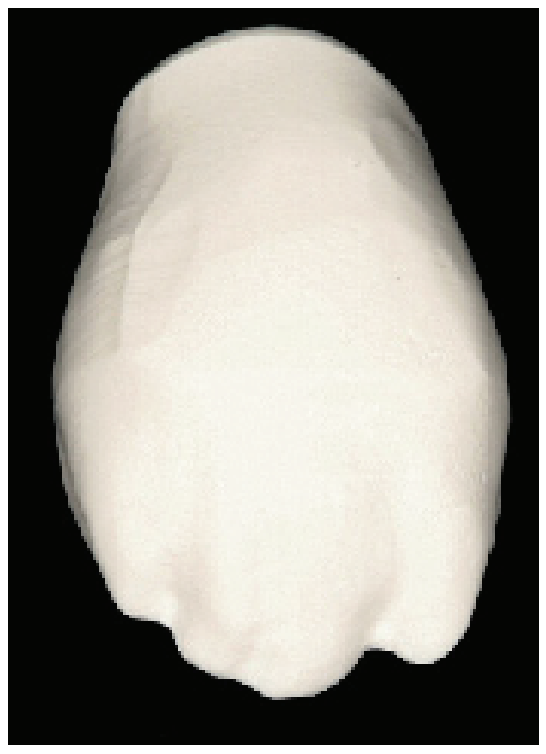

Figure 18: The milled crown before sintering.

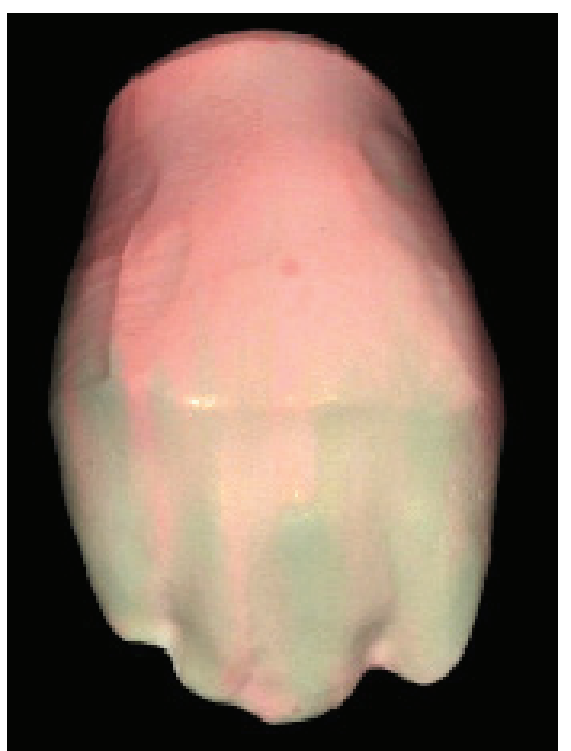

Figure 19: The milled was painted to match the patient's teeth. a)

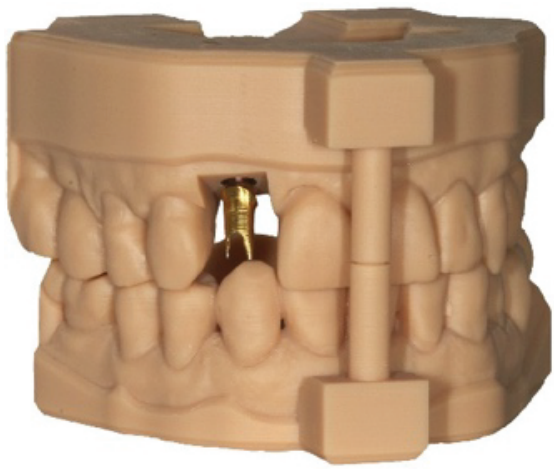

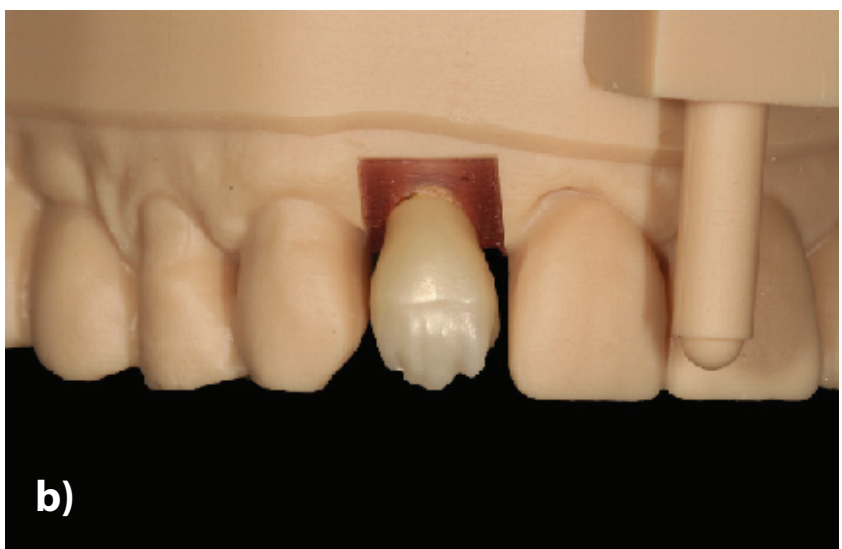

b)

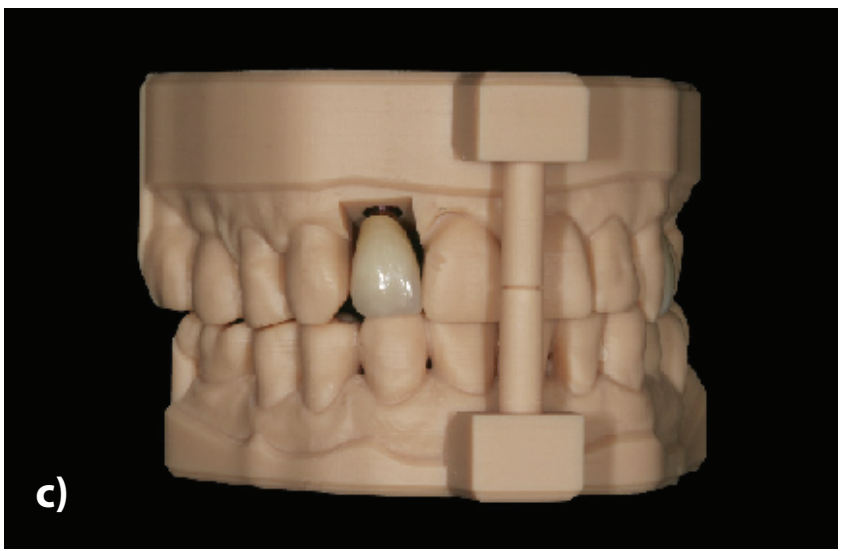

Figure 20: a) Atlantis abutment with printed model.

b) Sintered zirconium oxide (cut-back) crown on the model.

c) Finished zirconium oxide crown. 
Later-as in this case-it is used to secure a temporary crown with a prefabricated tooth, for example, or a self-made crown as a foundation for the long-term temporary restoration (Figures 10 and 11).

\section{Patient Case: In the Laboratory \\ Manufacture of CAD/CAM abutments}

The data from the intraoral scanner and all necessary patient information were available in the laboratory. After the data set was refined and an (optional) digital wax-up constructed, the digital file was loaded onto the Atlantis platform (Atlantis, Dentsply Sirona). The individual abutment was designed here (Virtual Abutment Design) on the basis of the ideal crown shape and with consideration of parameters such as the surrounding teeth and soft tissue anatomy (Figures 1216). The virtual draft design was then sent to the dental laboratory for inspection. During the approval process, the 3D editor can be used to modify the design of the abutment. Once the CAD design was approved, the abutment was manufactured from gold-shaded titanium coated with Titanium Nitride (TiN). The titanium nitride coating can be used to enhance the esthetic result, especially in the anterior region. While pure titanium can potentially create a gray shimmer in the area of the gingiva, the warm golden hue of the titanium nitride coating promotes a natural appearance.

\section{Final prosthetic restoration}

While the Atlantis abutment was produced by the Sirona manufacturer (Dentsply), the laboratory focused on the implant crown. The core file (abutment design) was successfully imported into the laboratory's proprietary CAD software. This was used to design a cutback crown, which is characterized by the use of individual parameters for determining the thickness of the ceramic veneer (Figure 17). The physical model essential for ceramic veneering was manufactured by Atlantis using a $3 \mathrm{D}$ printer. The crown was milled from a relatively opaque zirconium oxide material (Figure 18). To achieve vibrant coloration and a natural photo-optical effect "from within," the crown was customized with stains to match the patient's teeth before sintering (Figure 19). Manual ceramic veneering was performed to match the previously determined shade of the existing teeth. The abutment and crown had now been supplied by the manufacturing center. The crown fit precisely and was forwarded to the clinic after veneering with the abutment (Figure 20).

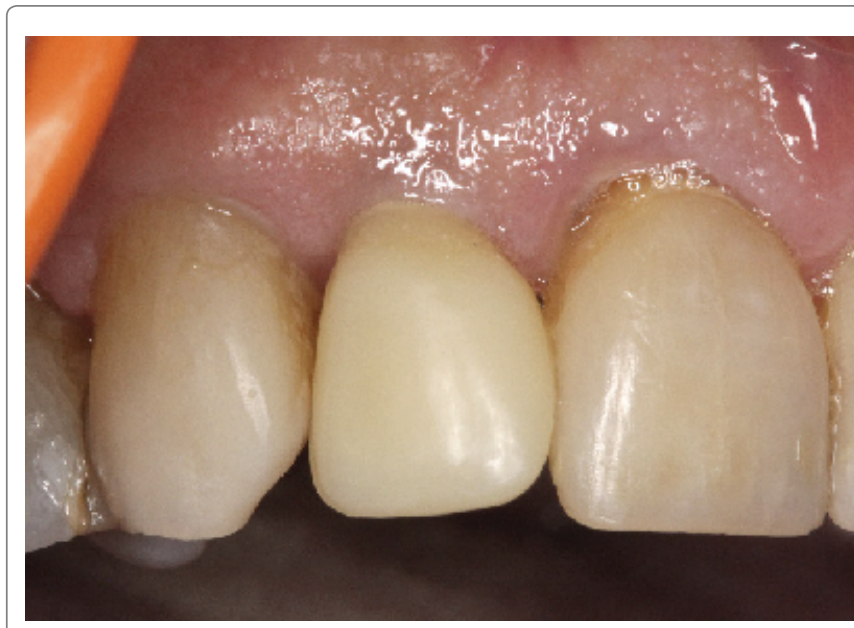

Figure 21: Temporary restoration shortly before removal.

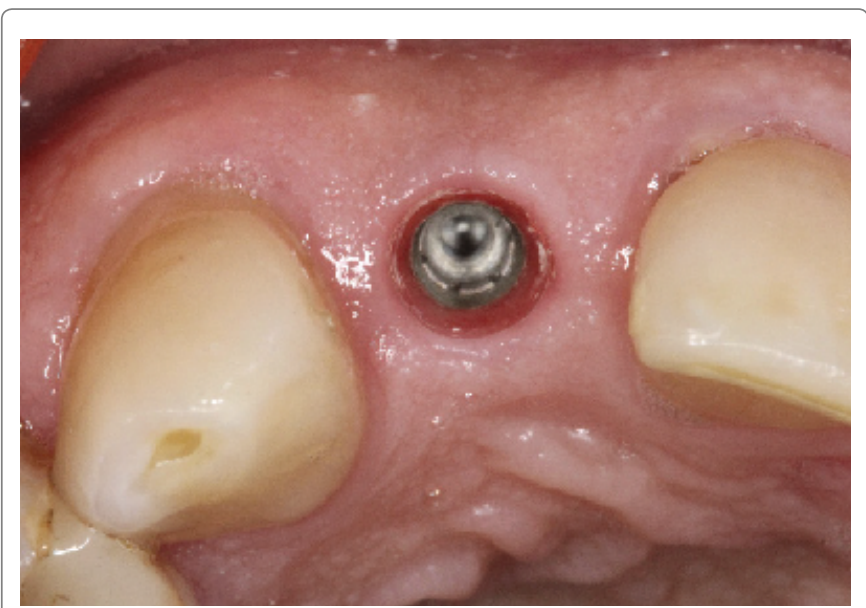

Figure 22: Clinical situation (healthy contoured peri-implant soft tissue) before inserting the abutment.
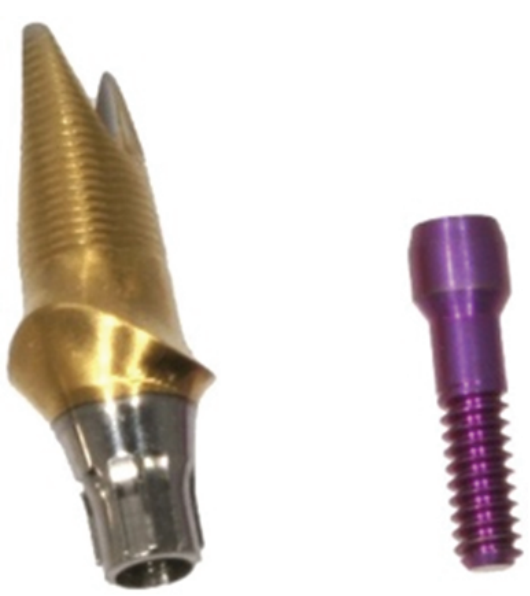

Figure 23: Atlantis abutment with the matching new screw for attachment to the implant.

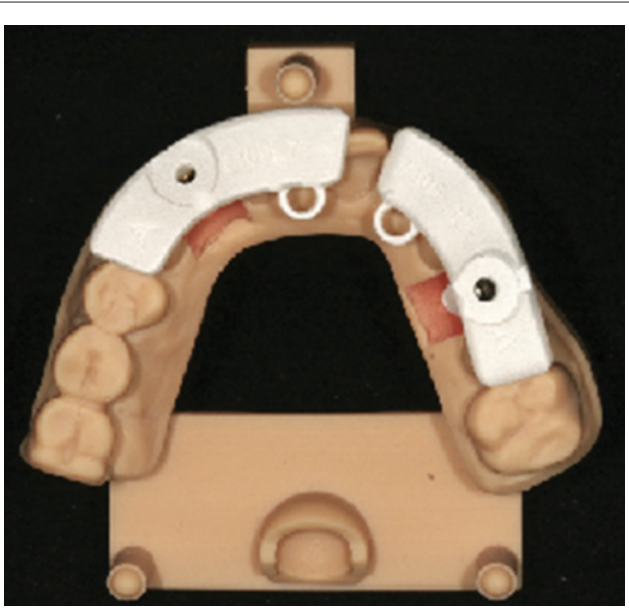

Figure 24: The abutments are attached on the printed model using the laboratory screw and the printed insertion keys are mounted. 


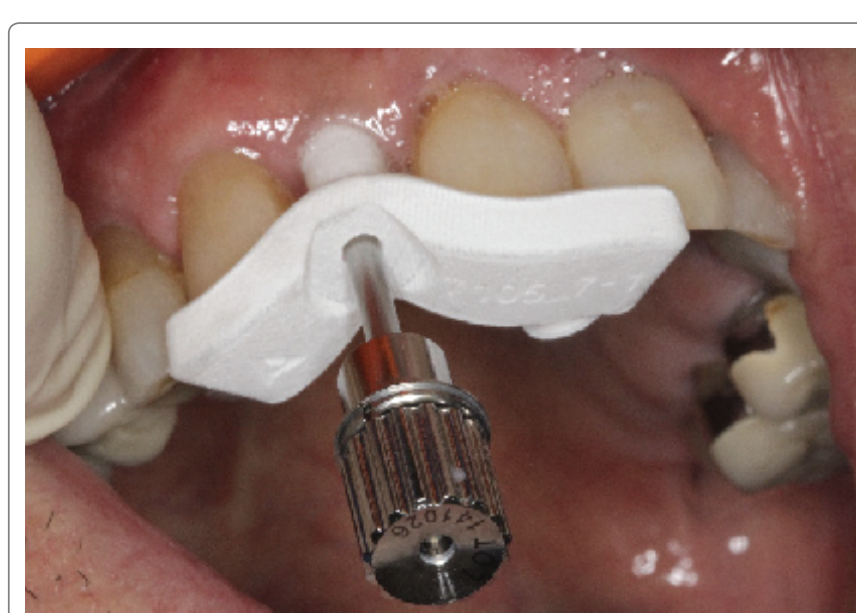

Figure 25: Inserting the abutment with a special plastic insert key.

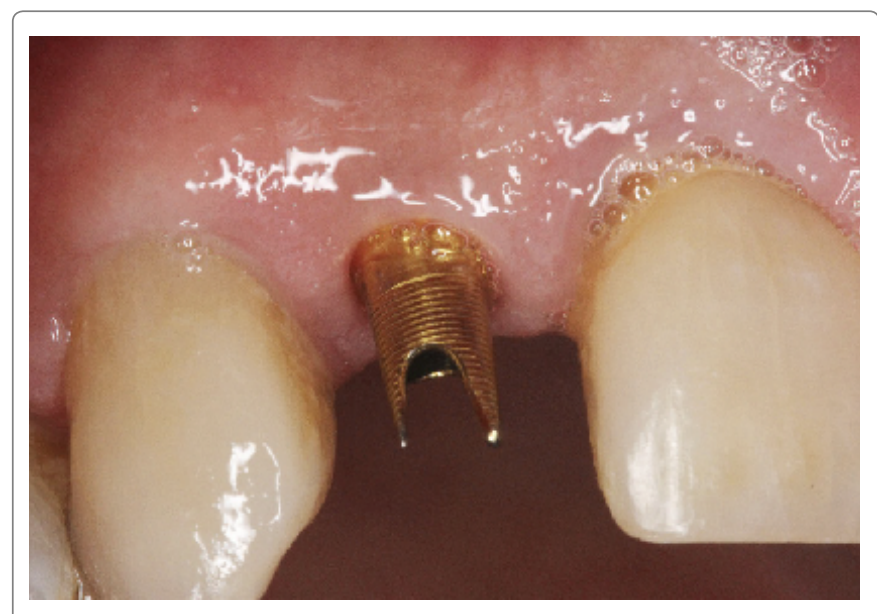

Figure 26: The patient-specific Atlantis abutment (material: titanium nitride-coated, GoldHue).

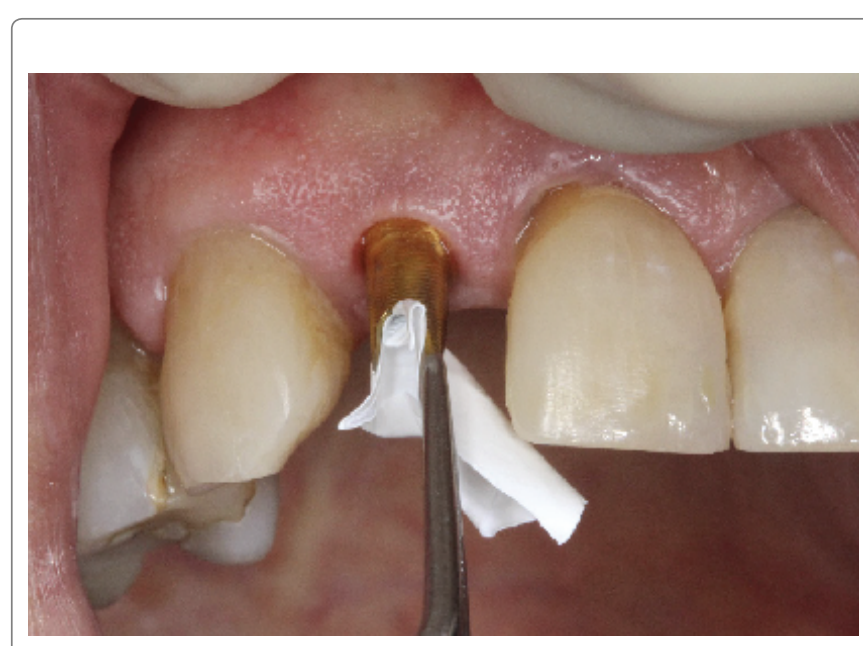

Figure 27: Sealing the screw access hole with Teflon tape.

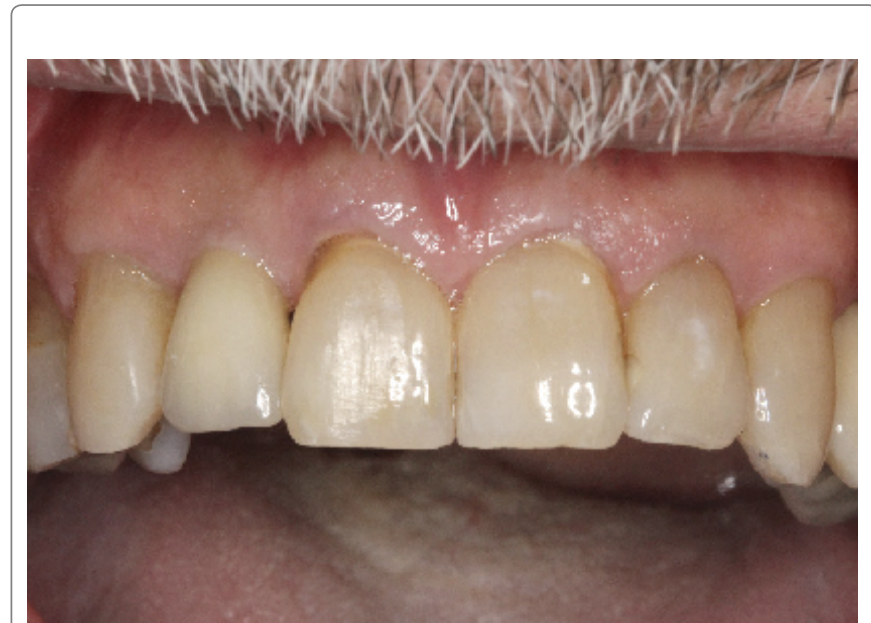

Figure 28: Clinical situation immediately after the all-ceramic crown was installed.

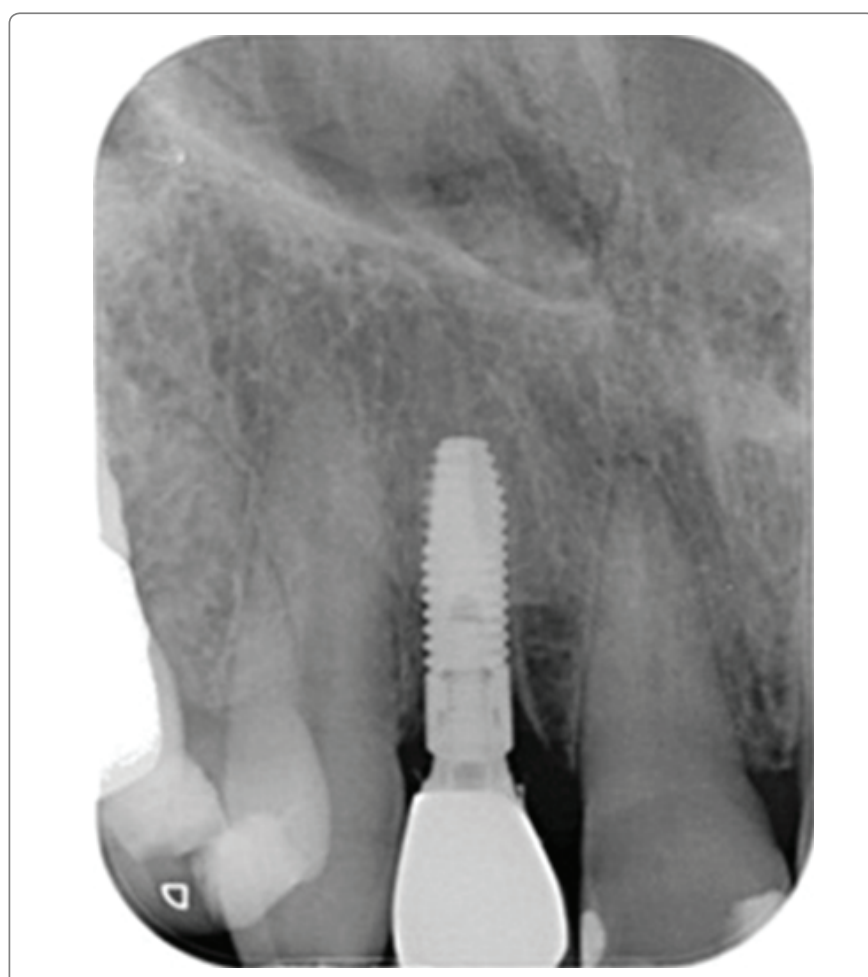

Figure 29: Follow-up X-ray image after inserting the implant-supported prosthetic restoration.

\section{Completion of the Restoration}

The temporary restoration (Figure 21) was removed. A healthy soft-tissue situation was observed (Figure 22). A detailed inspection confirmed stable peri-implant conditions and an osseointegrated implant in position 12. The abutment is supplied to the clinic with the abutment screw included separately to prevent the screw coming loose following its insertion in the laboratory (Figure 23). For transport to the clinic, the abutments were inserted into the model with the laboratory screws and the insertion keys were attached (Figure 24). 

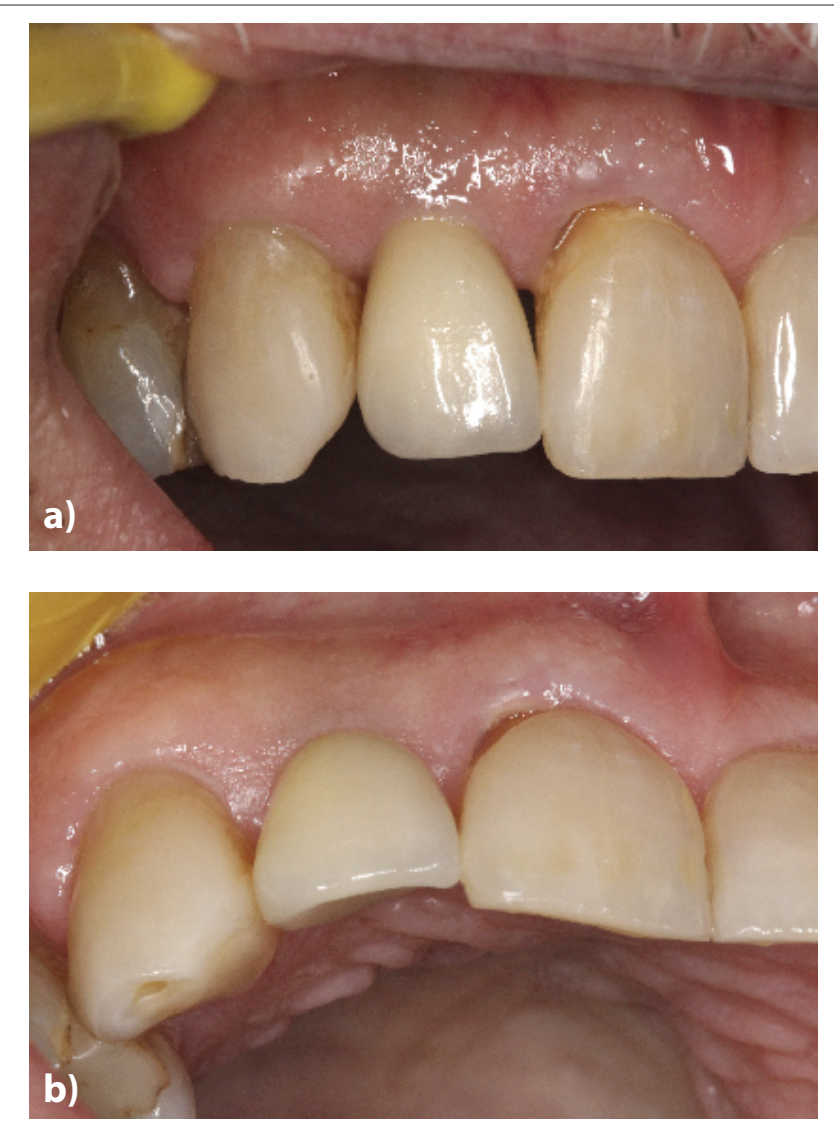

Figure 30: a) Seven days after fitting. The all-ceramic crown blends in seamlessly.

b) The peri-implant tissue appears healthy and natural.

Screwing the abutment to the implant was easy and safe with the special transfer key (Atlantis Insertion Guide, AIG) (Figure 25). First, the transfer key was placed on the adjacent teeth and the correct fit was verified. The key should fit firmly and securely to ensure accurate placement in the implant.

The abutment was then inserted into the Atlantis Insertion Guide. A slight resistance indicated that it was correctly positioned. After the abutment had been connected to the implant (Figure 26), the key was removed, the screw access hole sealed with Teflon tape (Figure 27 ), and the all-ceramic crown mounted (Figure 28). The implant crown slotted perfectly into the dental arch. A follow-up X-ray image confirmed the correct fit (Figure 29) and also revealed possible cement residue; a radiopaque material (e.g. Temp-Bond) should therefore be used. The X-ray taken after the prosthetic restoration also documents the initial position of the interproximal bone [16]. Following a clear and coherent plan, it was possible to replace tooth 12 promptly with an implant-supported prosthetic restoration (Figure 30).

\section{Conclusion}

Using the new Astra Tech Implant EV for immediate implant placement does not require any change in the day-to-day work of the surgeon, prosthodontist, or dental technician. The clinical and prosthetic protocols remain the same; only the thread design in the apical portion of the implant body was adapted. The tapering and the deeper thread incisions ensure greater primary stability, providing the foundation for safe immediate implant placement. The dentist benefits from a simplified procedure for implantation into fresh extraction sockets and under prepared osteotomies.

The Astra Tech Implant EV-a seemingly small change for a big advantage in routine clinical practice: Increased safety in immediate implant placements.

\section{References}

1. Lang NP, Pun L, Lau KY, Li KY, Wong MC (2012) A systematic review on survival and success rates of implants placed immediately into fresh extraction sockets after at least 1 year. Clin Oral Implants Res 5: 39-66.

2. Mangano C, Raes F, Lenzi C, Eccellente T, Ortolani M, et al. (2017) Immediate Loading of Single Implants: A 2-Year Prospective Multicenter Study. Int J Periodontics Restorative Dent 37: 69-78.

3. Nevins M, Wang HL (2019) Implant Therapy. Clinical Approaches and Evidence of Success. $2^{\text {nd }}$ Edition, Quintessence Publishing, USA 150.

4. Noelken R, Geier J, Kunkel M, Jepsen S, Wagner W (2018) Influence of soft tissue grafting, orofacial implant position, and angulation on facial hard and soft tissue thickness at immediately inserted and provisionalized implants in the anterior maxilla. Clin Implant Dent Relat Res 20: 674-682.

5. Currie F, Tilly C, Falk J (2019) Different apical implant designs and the impact on initial stability (P-BR-147). Clin Oral Impl Res 30: 174.

6. Ellingsen JE, Johansson CB, Wennerberg A, Holmén A (2004) Improved retention and bone-tolmplant contact with fluoridemodified titanium implants. Int J Oral Maxillofac Implants 19: 659-666.

7. Geckili O, Bilhan H, Bilgin T (2009) A 24-week prospective study comparing the stability of titanium dioxide grit-blasted dental implants with and without fluoride treatment. Int J Oral Maxillofac Implants 24: 684-688.

8. Berglundh T, Abrahamsson I, Albouy JP, Lindhe J (2007) Bone healing at implants with a fluoride-modified surface: an experimental study in dogs. Clin Oral Implants Res 18: 147-152.

9. Harder S, Dimaczek B, Açil Y, Terheyden H, Freitag-Wolf S, et al. (2010) Molecular leakage at implant-abutment connection-in vitro investigation of tightness of internal conical implant-abutment connections against endotoxin penetration. Clin Oral Investig 14: 427-432.

10. Toia M, Galli S, Cecchinato D, Wennerberg A, Jimbo R (2019) Clinical Evidence of OsseoSpeed EV Implants: A Retrospective Study and Characterization of the Newly Introduced System. Int J Periodontics Restorative Dent 39: 863-874.

11. Lee DW, Choi YS, Park KH, Kim CS, Moon IS (2007) Effect of microthread on the maintenance of marginal bone level: a 3-year prospective study. Clin Oral Implants Res 18: 465-470.

12. Hansson S (2003) A conical implant-abutment interface at the level of the marginal bone improves the distribution of stresses in the supporting bone. An axisymmetric finite element analysis. Clin Oral Implants Res 14: 286-293.

13. Abrahamsson I, Berglundh T, Wennström J, Lindhe J (1996) The peri-implant hard and soft tissues at different implant systems. A comparative study in the dog. Clin Oral Implants Res 7: 212-219.

14. Moon IS, Berglundh T, Abrahamsson I, Linder E, Lindhe J (1999) The barrier between the keratinized mucosa and the dental implant. An experimental study in the dog. J Clin Periodontol 26: 658-663.

15. Greenstein G, Cavallaro J (2017) Implant Insertion Torque: Its Role in Achieving Primary Stability of Restorable Dental Implants. Compend Contin Educ Dent 38: 88-95.

16. Wolfart S (2017) Implantatprothetik. EinPatientenorientiertesKonzept. Quintessenz Verlag, 396. 